EPSC Abstracts

Vol. 14, EPSC2020-569, 2020

https://doi.org/10.5194/epsc2020-569

Europlanet Science Congress 2020

(C) Author(s) 2020. This work is distributed under

the Creative Commons Attribution 4.0 License.

\title{
Follow-up of the activity and composition of the interstellar comet 2I/Borisov with MUSE
}

Cyrielle Opitom ${ }^{1,2}$, Michele Bannister ${ }^{3}$, Philippe Rousselot ${ }^{4}$, Alan Fitzsimmons ${ }^{5}$, Aurélie GuilbertLepoutre $^{6}$, Emmanuel Jehin ${ }^{7}$, Matthew Knight ${ }^{8}$, Youssef Moulane ${ }^{1,7,9}$, Meg Schwamb ${ }^{5}$, Darryl Seligman $^{10}$, Colin Snodgrass ${ }^{2}$, Bin Yang ${ }^{1}$, and the the 2I MUSE Observation Team*

${ }^{1}$ European Southern Observatory, Santiago, Chile (copitom@eso.org)

${ }^{2}$ Institute for Astronomy, University of Edinburgh, Royal Observatory, Edinburgh EH9 3HJ, UK

${ }^{3}$ School of Physical and Chemical Sciences -- Te Kura Matu, University of Canterbury, Private Bag 4800, Christchurch 8140, New Zealand

${ }^{4}$ Institut UTINAM UMR 6213, CNRS, Univ. Bourgogne Franche-Comté, OSU THETA, BP 1615, 25010 Besançon Cedex, France

${ }^{5}$ Astrophysics Research Centre, School of Mathematics and Physics, Queen's University Belfast, Belfast BT7 1NN, UK

${ }^{6}$ Univ Lyon, Univ Lyon 1, ENSL, CNRS, LGL-TPE, F-69622, Villeurbanne, France

${ }^{7}$ Space sciences, Technologies \& Astrophysics Research (STAR) Institute, University of Liège, Belgium

${ }^{8}$ Department of Physics, United States Naval Academy, 572C Holloway Rd, Annapolis, MD 21402, USA

${ }^{9}$ Oukaimeden Observatory, High Energy Physics \& Astrophysics Laboratory, Cadi Ayyad University

${ }^{10}$ Dept. of the Geophysical Sciences, University of Chicago, Chicago, IL 60637, USA

${ }^{*}$ A full list of authors appears at the end of the abstract

The interstellar comet 2I/Borisov was discovered on August 20, 2019. It is only the second interstellar object to be observed crossing our Solar System, and the first one for which outgassing was detected directly [1]. Early observations indicated that 2I/Borisov is depleted in $\mathrm{C}_{2}$, similarly to about $30 \%$ of Solar System comets $[2,3]$. Preliminary observations with the MUSE IFU performed in November 2019 confirmed that 2I is depleted in $\mathrm{C}_{2}$ but also showed it is rich in $\mathrm{NH}_{2}$ [4]. We present here results from the full observing campaign performed with the MUSE instrument.

MUSE is a multi-unit integral field spectrograph mounted on the UT4 telescope of the VLT [5]. The instrument covers the wavelength range from 480 to $930 \mathrm{~nm}$ with a resolving power of about 3000 . It has a large field of view of $1^{\prime} \times 1^{\prime}$ and a spatial resolution of $0.2^{\prime \prime}$, which makes it an ideal instrument to study extended sources. We observed 2I with MUSE on 16 different dates between November 14, 2019 and March 19, 2020. The observations started about one month before the perihelion passage and continued until the comet reached 3 au post-perihelion. This data sets constitutes a great opportunity to study the activity and coma composition of 2I over several months.

Our observations allow us to detect emission bands from $\mathrm{C}_{2}, \mathrm{NH}_{2}$, and $\mathrm{CN}$. Using a Haser model [6] we derive production rates for those 3 species and follow their evolution. We also study the evolution of the ratio between those production rates, to monitor how the composition of 2I coma changes as a function of time and distance from the Sun.

\section{References:}


[1] Fitzsimmons et al., 2019, The Astrophysical Journal Letters, Volume 885, Issue 1, article id.

L9, 6 pp.[2] Opitom et al., 2019, Astronomy \& Astrophysics, Volume 631, id.L8, 5 pp.; [3] Lin et al., 2019, The Astrophysical Journal Letters, Volume 889, Issue 2, id.L30;[4] Bannister et al, 2020, submitted to ApJ Letters; [5] Bacon et al, 2010, Proceedings of the SPIE, Volume 7735, id. 773508; [6] Haser, 1957,Bulletin de la Classe des Sciences de I'Académie Royale de Belgique, vol. 43, p. 740-750

the 2I MUSE Observation Team: The 2I MUSE Observation Team 